\title{
Experimental Evidence for Oxygen Sublattice Control in Polar Infinite Layer $\mathrm{SrCuO}_{2}$
}

\author{
D. Samal, ${ }^{1}$ Haiyan Tan, ${ }^{2}$ H. Molegraaf, ${ }^{1}$ B. Kuiper, ${ }^{1}$ W. Siemons, ${ }^{4}$ Sara Bals, ${ }^{2}$ Jo Verbeeck, ${ }^{2}$ Gustaaf Van Tendeloo, ${ }^{2}$ \\ Y. Takamura ${ }^{3}$ Elke Arenholz, ${ }^{5}$ Catherine A. Jenkins, ${ }^{5}$ G. Rijnders, ${ }^{1}$ and Gertjan Koster ${ }^{1}, *$ \\ ${ }^{1}$ MESA+ Institute for Nanotechnology, University of Twente, Post Office Box 217, 7500AE Enschede, The Netherlands \\ ${ }^{2}$ EMAT, University of Antwerp, Groenenborgerlaan 171, B-2020 Antwerp, Belgium \\ ${ }^{3}$ Department of Chemical Engineering and Materials Science, University of California-Davis, Davis, California 95616, USA \\ ${ }^{4}$ Materials Science and Technology Division, Oak Ridge National Laboratory, Oak Ridge, Tennessee 37831, USA \\ ${ }^{5}$ Advanced Light Source, Lawrence Berkeley National Laboratory, 1 Cyclotron Road, MS6R2100, Berkeley, California 94720, USA
}

(Received 3 May 2013; published 27 August 2013; publisher error corrected 28 August 2013)

\begin{abstract}
A recent theoretical study [Phys. Rev. B 85, 121411(R) (2012)] predicted a thickness limit below which ideal polar cuprates turn nonpolar driven by the associated electrostatic instability. Here we demonstrate this possibility by inducing a structural transformation from the bulk planar to chainlike structure upon reducing the $\mathrm{SrCuO}_{2}$ repeat thickness in $\mathrm{SrCuO}_{2} / \mathrm{SrTiO}_{3}$ superlattices with unit-cell precision. Our results, based on structural investigation by $\mathrm{x}$-ray diffraction and high resolution scanning transmission electron microscopy, demonstrate that the oxygen sublattice can essentially be built by design. In addition, the electronic structure of the chainlike structure, as studied by x-ray absorption spectroscopy, shows the signature for preferential hole occupation in the $\mathrm{Cu} 3 d_{3 z^{2}-r^{2}}$ orbital, which is different from the planar case.
\end{abstract}

DOI: 10.1103/PhysRevLett.111.096102

PACS numbers: 68.37.Ma, 78.70.Dm, 73.21.Cd

Atomic engineering of complex oxide thin films is now reaching a new paradigm: the possibility to control and measure the structure of the oxygen sublattice. The oxygen sublattice in oxide systems plays a pivotal role in determining the underlying structure, chemical bonding, and structure-property correlation. In the case of perovskite oxide materials, known for their wide range of useful properties and as promising candidates for novel electronic applications, the following situation is often encountered: an ionic thin film with a crystal structure consisting of alternating layers of nominally opposite charge grown on a substrate with nominally charged neutral layers produces a dipole and associated electrostatic field [1,2]. In such polar systems, the electrostatic energy due to the existing dipole layer is dependent on the film thickness and could counter the stabilizing effect of epitaxial strain. When the electrostatic energy is too large, the thin film system responds by redistributing the charge to neutralize the dipole $[1,2]$, which ultimately results in altered properties at the interface [1-6]. Various charge redistribution mechanisms can be at play: charge compensation by nonstoichiometry, adsorption of foreign atoms or ions, electronic charge redistribution, and, finally, structural transformation by atomic rearrangement [7-10]. In particular, the theoretical studies predict that the polarity of nanoscale objects and ultrathin films can be avoided by a structural transformation [8-11].

The infinite layer $\mathrm{SrCuO}_{2}$ (SCO) is an example of a polar system [12] and is widely known to be a parent structure for high- $T_{c}$ cuprate superconductors [13-15]. Its structure can be considered as an oxygen deficient perovskite, with $\mathrm{Cu}^{2+}$ ions coordinated to four planar $\mathrm{O}^{2-}$ ions and no $\mathrm{O}^{2-}$ ions in the $\mathrm{Sr}^{2+}$ plane [Fig. 1(a)].
These alternating layers with nominal charge of $\pm 2 e$ (in a purely ionic model) cause a buildup of electrostatic energy of $\sim 8 \mathrm{eV}$ per unit-cell (uc) of SCO. This value constitutes a strong electrostatic instability and makes the infinite layer structure highly polar. For very thin films of such materials, electronic charge transfer, which is a monotonic function of film thickness, as has been proposed by Zhong et al. [10] does not appear to be sufficient to quench the electrostatic instability. Surprisingly, a structural transformation involving the displacement of lighter atoms turns out to be the preferred mechanism. The study by Zhong et al. [10] on infinite layer cuprates predicts a transformation from the bulk planar to a chainlike structure for ultrathin films of about 5 uc thick. The resulting chainlike structure incorporates an $\mathrm{O}^{2-}$ ion in the $\mathrm{Sr}^{2+}$-plane as opposed to the planar structure and thus consists of charge neutral $\mathrm{SrO}^{0}$ and $\mathrm{CuO}^{0}$ layers [Fig. 1(b)]. The movement of an $\mathrm{O}^{2-}$ ion to the $\mathrm{Sr}^{2+}$-plane reduces the anisotropy of
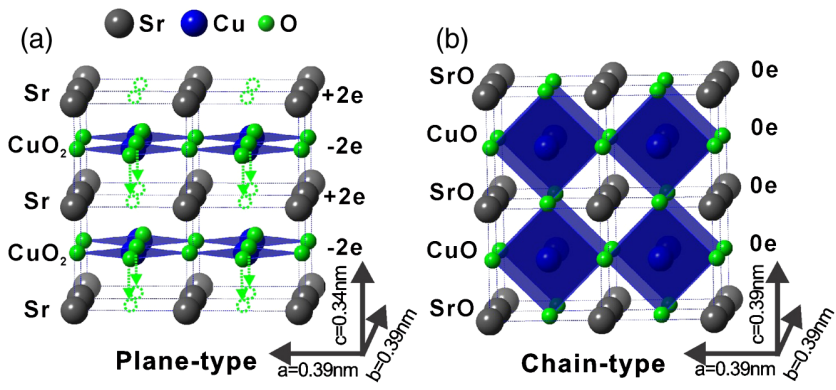

FIG. 1 (color online). Schematics of the (a) planelike and (b) chainlike crystal structures for tetragonal SCO. For clarity, we indicate the oxygen displacement by arrows in (a) that transform the planelike structure into chainlike one. 
the structure and acts as an apical center for the $\mathrm{Cu}^{2+}$ ions and thus offers the possibility for realizing the hole characteristic at $\mathrm{Cu} 3 d_{3 z^{2}-r^{2}}$ orbital as demonstrated by us using polarized x-ray absorption spectroscopy (XAS). Besides the aforementioned phenomena, Aruta et al., [16] hypothesize on another mechanism via oxygen redistribution in the interfacial planes, which could alleviate the built-in potential without any electronic redistribution, to explain their experimental results using hard x-ray photoelectron spectroscopy on $\mathrm{CaCuO}_{2} / \mathrm{SrTiO}_{3}$ superlattices (SLs) prepared under different oxidizing conditions.

In this Letter, we show that an atomic rearrangement of oxygen ions occurs within the bulk of the layers of SCO below a certain thickness. We study $\mathrm{SCO} / \mathrm{SrTiO}_{3}$ (STO) SLs grown by pulsed laser deposition (PLD). In situ monitoring with reflective high-energy electron diffraction (RHEED) allows us to control the thickness of SLs with unit-cell precision. Our results based on structural and spectroscopic signatures unambiguously demonstrate a transformation from planar to chainlike structure in ultrathin films of SCO. Besides being an interesting model system to study such dramatic structural changes and their formation pathways, we demonstrate that the oxygen sublattice can be effectively engineered by systematic variation of the individual sub-layer thicknesses in the SL, giving each layer a specific function, for example, current-carrying layers, charge reservoirs, and scaffolding layers.

All the SLs were grown on $\mathrm{TiO}_{2}$-terminated [17] (001)oriented STO substrates by reflection high-energy electron diffraction (RHEED) assisted PLD. The deposition conditions have been optimized to result in the growth of the infinite layer tetragonal phase of SCO (see Supplemental Material, Fig. S1) [18]. The structural characterization was carried out using $\mathrm{x}$-ray diffraction, high-resolution scanning transmission electron microscopy (STEM) and composition by electron energy loss spectroscopy (EELS). High-angle annular dark-field (HAADF), annular bright field (ABF) STEM images and EEL spectra were acquired using a FEI $\operatorname{Titan}^{3} 80-300$, equipped with a double aberration corrector. The spatial resolution of the STEM images is $1 \AA$ and the energy resolution of the EELS spectra is $1.1 \mathrm{eV}$. The ABF/ $\mathrm{ADF}$ images are recorded with an annular detector, capturing scattering from 26 to 60 and from 15 to $24 \mathrm{mrad}$, respectively. The image simulations [inset in Fig. 4] are obtained using STEMSIM [19] and considering a supercell consisting of $5 \times 5$ unit-cells with a total of $180 \times 180$ pixels. Polarized XA spectra were acquired at beam line 4.0.2 at the Advanced Light Source in total electron yield mode by monitoring the sample drain current.

We first present the results of the structural investigation of two types of SLs: (i) $\left[(\mathrm{SCO})_{m} /(\mathrm{STO})_{2}\right]_{20}$ where $m$ equals the number of unit cells of SCO that varies from 3-16 and (ii) a hybrid structure consisting of SCO layers of different thicknesses: $\left[(\mathrm{SCO})_{8} /(\mathrm{STO})_{2} /(\mathrm{SCO})_{3} /(\mathrm{STO})_{2}\right]_{10}$. In Figs. 2(a) and 2(b) we show the $\theta-2 \theta$ x-ray diffraction
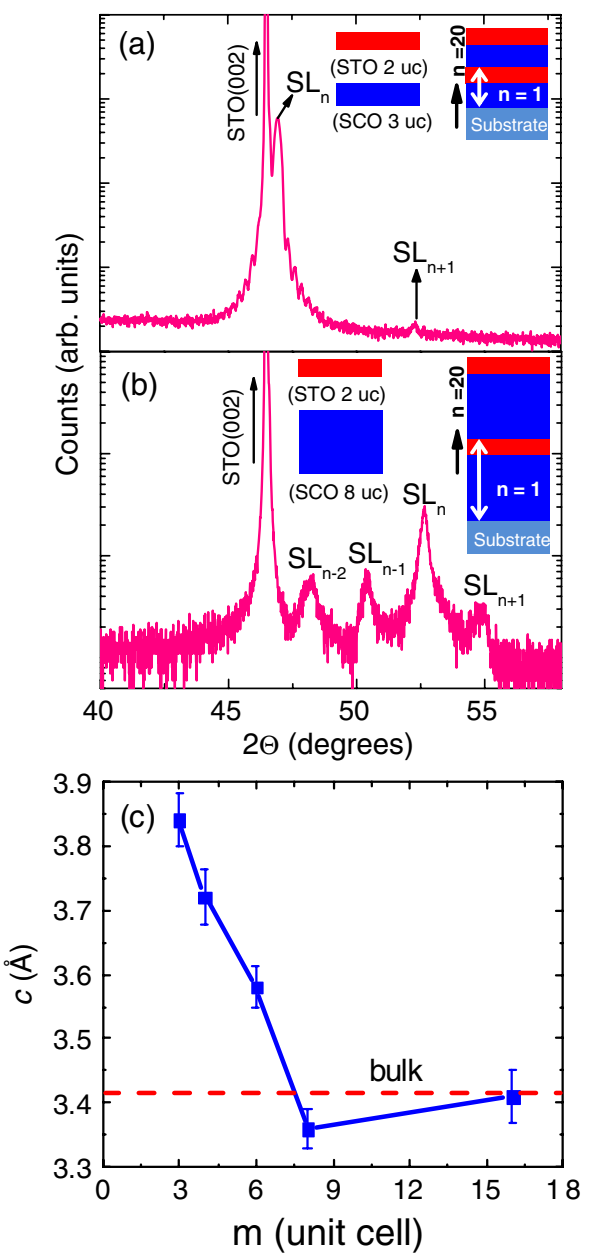

FIG. 2 (color online). (a) $\theta-2 \theta$ XRD spectra of (a) $\left[(\mathrm{SCO})_{3} /(\mathrm{STO})_{2}\right]_{20}$ and (b) $\left[(\mathrm{SCO})_{8} /(\mathrm{STO})_{2}\right]_{20}$ SLs grown on (001)-oriented STO. The intense peaks belong to the STO substrate, and peaks indexed by SL refer to the satellite peaks from the SL structure. (c) Average $c$-axis lattice parameter as a function of SCO thickness for $\left[(\mathrm{SCO})_{m} /(\mathrm{STO})_{2}\right]_{20}$ SLs. The horizontal dashed line marks the reported bulk $c$-axis lattice parameter for infinite layer SCO.

(XRD) patterns for two representative SLs: $\left[(\mathrm{SCO})_{3} /\right.$ $\left.(\mathrm{STO})_{2}\right]_{20}$ and $\left[(\mathrm{SCO})_{8} /(\mathrm{STO})_{2}\right]_{20}$. A signature of satellite peaks corresponding to the SL structure is observed in both cases, which demonstrates the structural quality of the SLs. The $\left[(\mathrm{SCO})_{3} /(\mathrm{STO})_{2}\right]_{20}$ SL also displays distinct finite-size oscillations arising from the finite thickness of the film, which are absent in the $\left[(\mathrm{SCO})_{8} /(\mathrm{STO})_{2}\right]_{20}$ SL. As noticed from the RHEED spectrum and the atomic force microscopy (AFM) images, the overall film roughness increases with increasing SCO layer thickness (see Supplemental Material, Fig. S2 [18]). Apparently, 2 uc of STO is not sufficient to repair the roughness of the preceding SCO layers. The average $c$-axis lattice parameter estimated from the zeroth order satellite peak positions is plotted as a function of the SCO thickness $(m)$ in the SLs in Fig. 2(c) and provides key information about the arrangement of 
oxygen ions surrounding the $\mathrm{Cu}$ ions in the SCO films. A clear trend of decreasing average $c$-axis lattice parameter is observed as $m$ increases, ranging from 3.84 to $3.36 \AA$ for $\left[(\mathrm{SCO})_{3} /(\mathrm{STO})_{2}\right]_{20}$ and $\left[(\mathrm{SCO})_{8} /(\mathrm{STO})_{2}\right]_{20}$, respectively. Most importantly, the average $c$-axis lattice parameter levels out at a value close to the expected bulk SCO value $(a=b=3.926 \AA$ and $c=3.432 \AA$ ) [20] for $m \geq 8$. The increase in the average $c$-axis lattice parameter at lower $m$ suggests that the SCO layers undergo a gradual structural transformation to the chainlike structure characterized by an elongation along the $c$ axis.

Further insight into the structural transformation was obtained by a detailed STEM study. Figure 3(a) shows a HAADF-STEM image of the SL $(m=3)$ in which the heavy atom columns show up as bright spots in the atomic resolution image. It can be seen that the $(\mathrm{STO})_{2}$ and $(\mathrm{SCO})_{3}$ layers grow epitaxially with respect to each other. Using the unit-cell parameter of the STO substrate $(a=3.905 \AA)$ as an internal calibration, the $c$-axis lattice parameters of both $(\mathrm{STO})_{2}$ and $(\mathrm{SCO})_{3}$ layers are precisely measured to be 3.83(3) $\AA$. This is in good agreement to the value of average $c$-axis lattice parameter for $(\mathrm{SCO})_{3}$ layers from the XRD study. Note that the $c$-axis lattice parameter of STO in the SL is also slightly reduced with respect to the bulk STO value (3.905 $\AA$ ). When using HAADF-STEM, the intensity of the atomic columns scales with the atomic number $Z$ of the atoms present in the column [21]. In this case, the contrast difference between STO and SCO layers is limited due to the very similar atomic weight of the columns. Therefore, EELS was performed to confirm the chemical composition of the layers in a direct manner. The inset of Fig. 3(a) is a color map obtained from EELS showing the distribution of $\mathrm{Ti}$ and $\mathrm{Cu}$ signals across the film indicating the SCO and STO layers and confirms the designed SL structure with some intermixing of $\mathrm{Ti}$ into SCO. Detailed EELS analysis reveals that the Ti signal remains $4^{+}$and the oxygen content is reduced with respect to the STO layer. This is consistent with a picture of some intermixed octahedrally coordinated $\mathrm{Ti}$ atoms while the $\mathrm{Cu}$ ions remain planar coordinated in either plane or chain-type.

To investigate the $c$-axis lattice parameter variation with $\mathrm{SCO}$ thickness, the hybrid $\left[(\mathrm{SCO})_{8} /(\mathrm{STO})_{2} /(\mathrm{SCO})_{3} /\right.$ $\left.(\mathrm{STO})_{2}\right]_{10}$ SL was also investigated by STEM. Its SL structure model is sketched in Fig. 3(b) together with a HAADF-STEM image. The $c$-axis lattice parameter from the HAADF-STEM image for the $(\mathrm{SCO})_{8}$ layers is measured to be 3.43(3) $\AA$, which is $10 \%$ less than that of the $(\mathrm{SCO})_{3}$ layers [3.83(3) $\AA$ ] in the same film [Fig. 3(b)]. This clearly illustrates the distinction in relation to the $c$-axis lattice parameter between $(\mathrm{SCO})_{8}$ and $(\mathrm{SCO})_{3}$ layers and supports our XRD results. The inset of Fig. 3(b) is a color map obtained from EELS that shows the distribution of Ti and $\mathrm{Cu}$ signals across the film indicating the SCO and STO layers, in agreement with the as designed structure.
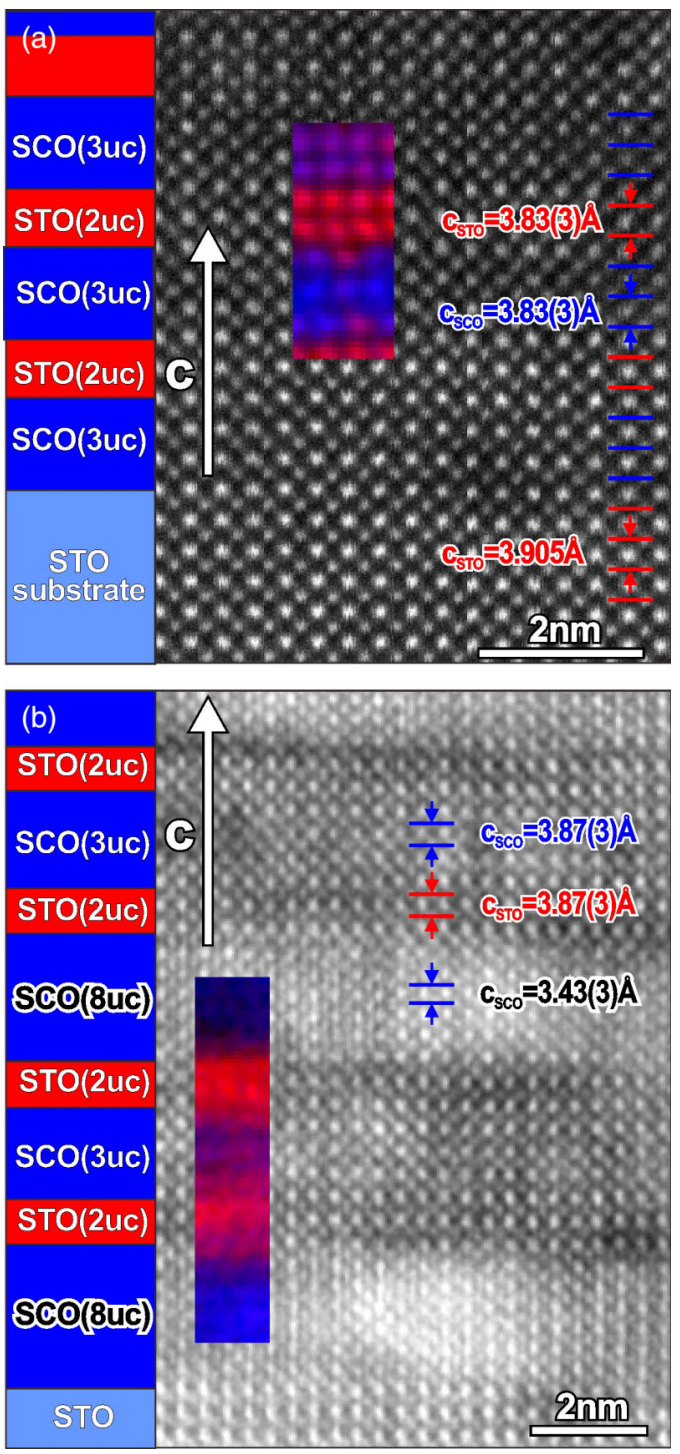

FIG. 3 (color online). HAADF-STEM image (with its SL structure sketched at the left side) for (a) $\left[(\mathrm{SCO})_{3} /(\mathrm{STO})_{2}\right]_{20}$ and (b) $\left[(\mathrm{SCO})_{8} /(\mathrm{STO})_{2} /(\mathrm{SCO})_{3} /(\mathrm{STO})_{2}\right]_{10}$, respectively. The insets are the low pass filtered elemental color map $[\mathrm{red}=\mathrm{Ti}$ (ash gray); blue $=\mathrm{Cu}$ (deep gray)] obtained with EELS and it agrees with the as-designed model. The $c$-axis lattice parameters estimated for different layers are indicated in the images.

Since the arrangement of the oxygen atoms is different for chain and planelike SCO layers, it is important to directly identify the oxygen ions on a local scale. ABFSTEM imaging collects the low angle scattered electrons as an alternative to HAADF-STEM, which collects electrons scattered to higher angles. As a consequence, ABF-STEM is more sensitive to the light atoms whereas HAADFSTEM mostly maps the heavy atoms [22]. Combining both techniques in a color map therefore gives a unique opportunity to monitor the positions of the oxygen atoms with respect to the heavy atoms. Such a superimposed color map is presented in Fig. 4 with the low angle scattering 


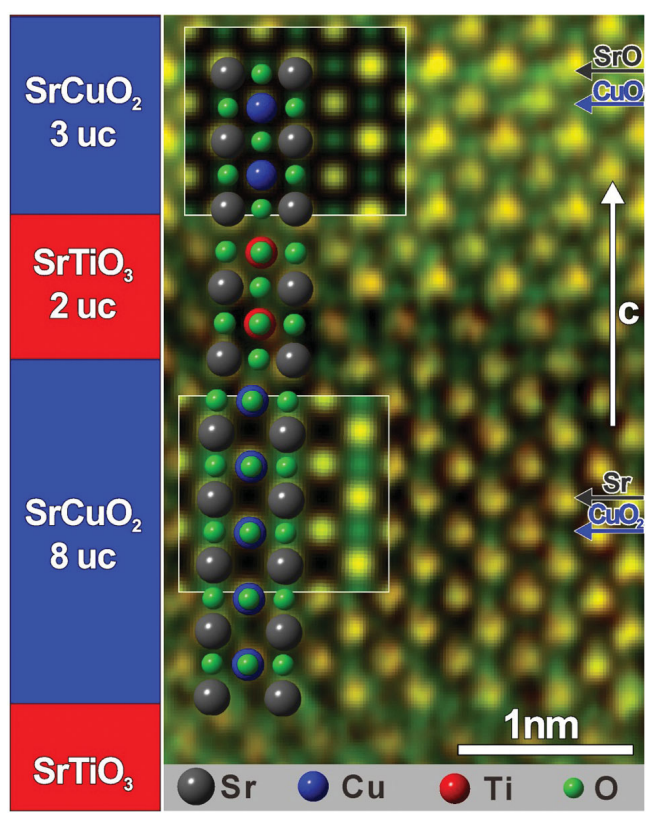

FIG. 4 (color). Combined color map from the simultaneously acquired $\mathrm{ADF}$ (red) and inverted intensity ABF image (green). The yellow peaks indicate the heavy elements $(\mathrm{Sr}, \mathrm{Cu}$, and $\mathrm{Ti}$ ) while the green peaks highlight the light elements $(\mathrm{O})$. A clear structural difference is seen between the $\mathrm{SCO}_{3}$ (chain) and the $\mathrm{SCO}_{8}$ (planar) layer, which is confirmed by comparing to simulated images (insets) in the respective layers. A structural model is overlaid.

events (ABF-STEM) in green and the high angle (HAADFSTEM) ones in red. The columns containing heavy elements $(\mathrm{Sr}, \mathrm{Cu}$, and $\mathrm{Ti})$ together with oxygen atoms are visible as yellow (overlay of red and green) dots whereas the columns containing only oxygen atoms are visible in green. We clearly observe the oxygen columns between all the heavy atomic columns in $(\mathrm{SCO})_{3}$ and $(\mathrm{STO})_{2}$ layers but no oxygen in the $\mathrm{Sr}$ planes of the $(\mathrm{SCO})_{8}$ layer. This directly confirms the planar-arrangement [Fig. 1(a)] of the oxygen atoms in the $(\mathrm{SCO})_{8}$ layer. However, the arrangement of the oxygen atoms in $(\mathrm{SCO})_{3}$ is in agreement with the chainlike structure as depicted in Fig. 1(b). Simulated images assuming these structures are shown to guide the reader in interpreting the subtle differences visible in Fig. 4. Thus, we clearly view a thickness-dependent structural transformation via oxygen-rearrangement throughout the bulk of the SCO layer unlike to a recent hypothesis by Aruta et al. [16] based on a rearrangement of oxygen ions only at the interface between $\mathrm{CaCuO}_{2}$ and STO.

In order to determine how the $\mathrm{Cu} 3 d$ orbital occupancy is modified by the structural transformation, we performed XA spectroscopy using linearly polarized $\mathrm{x}$ rays. By changing the direction of the X-ray polarization ( $\boldsymbol{E}$ vector) relative to the sample surface, one can probe the angular dependence of the empty valence states. In particular, the electronic transition intensity scales with the number of empty orbitals in the direction of $\boldsymbol{E}$. The linearly polarized

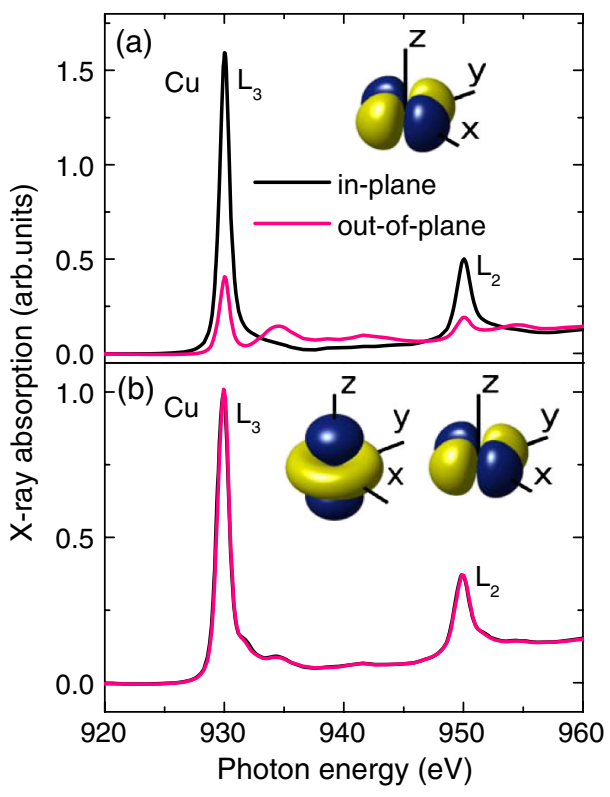

FIG. 5 (color online). Polarized XA spectra for (a) $\left[(\mathrm{SCO})_{8} /(\mathrm{STO})_{2}\right]_{5}$ and (b) $\left[(\mathrm{SCO})_{3} /(\mathrm{STO})_{2}\right]_{10}$ SLs. The insets show schematics for the $d$ orbitals where the hole is preferentially localized.

$\mathrm{x}$ rays were incident upon the sample with a $60^{\circ}$ angle relative to the sample normal, and the $\boldsymbol{E}$ vector was either in-plane or at a $30^{\circ}$ angle relative to the sample normal (i.e., the $c$ axis of the SL). To avoid charging effects during the measurement, the $\left[(\mathrm{SCO})_{3} /(\mathrm{STO})_{2}\right]_{10}$ and $\left[(\mathrm{SCO})_{8} /\right.$ $\left.(\mathrm{STO})_{2}\right]_{5}$ SLs were grown on $\mathrm{Nb}$-doped $(0.05 \%$ at.) STO substrates. Figure 5 shows the XA spectra at the $\mathrm{Cu} L_{2,3}$ edges for $\left((\mathrm{SCO})_{3} /(\mathrm{STO})_{2}\right)_{10}$ and $\left.(\mathrm{SCO})_{8} /(\mathrm{STO})_{2}\right)_{5}$ SLs with the $\boldsymbol{E}$ vector aligned in-plane and canted out-of-plane relative to the $c$ axis of the SL. For both SLs, the peaks at the $\mathrm{Cu} L_{2,3}$ edges (i.e., at about 930 and $950 \mathrm{eV}$ ) correspond to transitions from $\mathrm{Cu} 2 p_{3 / 2}$ to $\mathrm{Cu} 3 d$ and $\mathrm{Cu} 2 p_{1 / 2}$ to $\mathrm{Cu} 3 d$ orbitals respectively. These transitions are referred as $2 p^{6} 3 d^{9} \rightarrow 2 p^{5} 3 d^{10}$, where an electron from the $\mathrm{Cu} 2 p$ is promoted to the $\mathrm{Cu} 3 d$ orbital $[23,24]$. In Fig. 5(a), we observe a strong dependence of the spectral intensity on the polarization direction for the $\left[(\mathrm{SCO})_{8} /(\mathrm{STO})_{2}\right]_{5} \mathrm{SL}$, with a significantly higher absorption when $\boldsymbol{E}$ is aligned in-plane compared to when it cants out-of-plane. In addition to the main $\mathrm{Cu} L_{3}$ peak, a second significantly weaker peak is observed $\sim 4.6 \mathrm{eV}$ higher in energy. In previous studies on oxygen deficient $\mathrm{YBa}_{2} \mathrm{Cu}_{3} O_{\mathrm{x}}$, this peak has been related to a small content of monovalent $\mathrm{Cu}$ [25]. The stronger absorption for the inplane $\boldsymbol{E}$ vector indicates that most of the $\mathrm{Cu}$ sites have holes occupying the $d_{x^{2}-y^{2}}$ orbital. However, the scenario differs for $\left[(\mathrm{SCO})_{3} /(\mathrm{STO})_{2}\right]_{10}$ SL $[$ Fig. $5(\mathrm{~b})]$ due to the presence of apical oxygen. In this case, the peak intensity and spectral shape are found to be nearly identical irrespective of the orientation of the $\boldsymbol{E}$, an indication that the holes are distributed equally over the $d_{x^{2}-y^{2}}$ and $d_{3 z^{2}-r^{2}}$ 
orbitals. Because of the symmetry of such an angular distribution, the transition intensity is equal in all principal directions, as well as any angles between the principle axes, and no linear dichroism is observed. The $\mathrm{Cu}$ spectra are characterized by two weak peaks in addition to the main $\mathrm{Cu} L_{3}$ peak, namely the monovalent $\mathrm{Cu}$ peak and a shoulder at $\sim 1.4 \mathrm{eV}$ above the main peak, which is usually ascribed to the $3 d^{10} L$ final-state configuration, where $L$ denotes a $2 p$ hole at the oxygen sites [26]. It should be noted that there might be additional factors that contribute to the XA spectra, such as oxygen defects and/or the interfacial oxygen $[27,28]$, which can give rise to apical site for $\mathrm{Cu}$ ions. However, these secondary effects cannot fully account for such a marked difference.

In summary, we have experimentally shown a structural transformation (planar to chainlike) occurring via atomic reconstruction in ultrathin films of $\mathrm{SrCuO}_{2}$. The experiments follow the theoretical prediction of a strong electrostatic instability associated with the polar nature of layered cuprates that drives the system for atomic rearrangement. Our findings clearly reveal an increase of $c$-axis lattice parameter by $\sim 0.5 \AA$ and the presence of oxygen ions in the $\mathrm{Sr}$ plane for ultrathin $\mathrm{SrCuO}_{2}$ films contrary to its bulk counterpart. Combination of $\mathrm{ABF}$ and ADF imaging directly visualizes the change in position of the oxygen atoms. This observation is further complemented with polarized XAS experiments showing a clear distinction between the planar and chainlike structure with respect to hole distribution at $\mathrm{Cu}$ site. We believe that our finding will trigger activities to design novel cuprate heterostructures with alternation of chain and planelike layers (the basic building-blocks in cuprates) to look for high- $T_{c}$ superconductivity. As a final remark, we would like to point out that our finding of chainlike structure in ultrathickness limit could hold the key as to why ultrathin cuprates do not exhibit superconductivity.

This work was carried out with financial support from AFOSR and EOARD project (Project No. FA8655-10-13077) and also supported by funding from the European Research Council under the 7th Framework Program (FP7), ERC Grant No. 246791-COUNTATOMS and ERC Starting Grant No. 278510 VORTEX. The QuAnt-EM microscope was partly funded by the Hercules fund from the Flemish Government. This work was partially funded by the European Union Council under the 7th Framework Program (FP7) Grant No. NMP3-LA2010-246102 IFOX. The authors acknowledge financial support from the European Union under the Seventh Framework Program under a contract for an Integrated Infrastructure No. 312483-ESTEEM2. Advanced Light Source is supported by the Office of Science, Office of
Basic Energy Sciences of the U.S. Department of Energy (DOE) under Contract No. DE-AC02-05CH11231. Y.T. acknowledges support from the National Science Foundation (DMR-0747896). W. S. was supported by the US DOE, Basic Energy Sciences, Materials Sciences and Engineering Division. D.S. thanks Z. Zhong from Vienna University of Technology, Austria for scientific discussion.

*g.koster@utwente.nl

[1] A. Ohtomo and H. Y. Hwang, Nature (London) 427, 423 (2004).

[2] A. J. Millis and D. G. Schlom, Phys. Rev. B 82, 073101 (2010).

[3] J. Chakhalian et al., Science 318, 1114 (2007).

[4] N. Reyren et al., Science 317, 1196 (2007).

[5] L. Li et al., Nat. Phys. 7, 762 (2011).

[6] A. Brinkman et al., Nat. Mater. 6, 493 (2007).

[7] C. J. Noguera, Phys. Condens. Matter 12, R367 (2000).

[8] J. Goniakowski, C. Noguera, and L. Giordano, Phys. Rev. Lett. 98, 205701 (2007).

[9] J. Goniakowski, C. Noguera, and L. Giordano, Phys. Rev. Lett. 93, 215702 (2004).

[10] Z. Zhong, G. Koster, and P. J. Kelly, Phys. Rev. B 85, 121411(R) (2012).

[11] A. Harrison, E. A. Kraut, J. R. Waldrop, and R. W. Grant., Phys. Rev. B 18, 4402 (1978).

[12] G. Koster et al., J. Phys. Condens. Matter 20, 264007 (2008).

[13] G. E. S. Kikkawa et al., Physica (Amsterdam) 196C, 271 (1992).

[14] M. Azuma et al., Nature (London) 356, 775 (1992).

[15] M. G. Smith et al., Nature (London) 351, 549 (1991).

[16] C. Aruta et al., Phys. Rev. B 87, 155145 (2013).

[17] G. J.H. M. Rijnders et al., Appl. Phys. Lett. 70, 1888 (1997).

[18] See supplemental material at http://link.aps.org/ supplemental/10.1103/PhysRevLett.111.096102 for structural characterization by XRD and surface roughness by RHEED and AFM.

[19] A. Rosenauer and M. Schowalter, STEMSIM-a new software tool for simulation of STEM HAADF: Microscopy of Semiconducting Materials (MSM) Conference, 2007, Vol. 120 (Springer, Netherlands, 2008), p. 169.

[20] M. Takano et al., Physica (Amsterdam) 159C, 375 (1989).

[21] P. Hartel, H. Rose, and C. Dinges, Ultramicroscopy 63, 93 (1996).

[22] S. Findlay et al., Ultramicroscopy 110, 903 (2010).

[23] D. D. Sarma et al., Phys. Rev. B 37, 9784 (1988).

[24] C. T. Chen et al., Phys. Rev. Lett. 68, 2543 (1992).

[25] N. Nucker et al., Phys. Rev. B 51, 8529 (1995).

[26] M. Minola et al., Phys. Rev. B 85, 235138 (2012).

[27] D. Di Castro. et al., Phys. Rev. B 86, 134524 (2012).

[28] C. Aruta et al., Phys. Rev. B 78, 205120 (2008). 